\title{
Deciphering the underlying mechanisms of Diesun Miaofang in traumatic injury from a systems pharmacology perspective
}

\author{
CHUN-SONG ZHENG ${ }^{1 *}$, CHANG-LONG FU $^{1 *}$, CAI-BIN PAN ${ }^{1}$, HONG-JUAN BAO ${ }^{2}$, \\ XING-QIANG CHEN ${ }^{1}$, HONG-ZHI YE ${ }^{1}$, JIN-XIA YE ${ }^{1}$, GUANG-WEN WU ${ }^{1}$, \\ XI-HAI LI ${ }^{1}$, HUI-FENG XU ${ }^{1,3}$, XIAO-JIE XU ${ }^{1,4}$ and XIAN-XIANG LIU ${ }^{1}$ \\ ${ }^{1}$ Institute of Bone Disease, Academy of Integrative Medicine, Fujian University of Traditional Chinese Medicine, \\ Fuzhou, Fujian 350122; ${ }^{2}$ Department of Pharmacy, Xiamen Medical College, Xiamen, Fujian 361008; \\ ${ }^{3}$ Fujian Key Laboratory of Integrative Medicine on Geriatrics, Fujian University of Traditional Chinese Medicine, \\ Fuzhou, Fujian 350122; ${ }^{4}$ Beijing National Laboratory for Molecular Sciences, College of Chemistry and Molecular Engineering, \\ Peking University, Beijing 100871, P.R. China
}

Received July 6, 2014; Accepted March 18, 2015

DOI: $10.3892 / \mathrm{mmr} .2015 .3638$

\begin{abstract}
Diesun Miaofang (DSMF) is a traditional herbal formula, which has been reported to activate blood, remove stasis, promote qi circulation and relieve pain. DSMF holds a great promise for the treatment of traumatic injury in an integrative and holistic manner. However, its underlying mechanisms remain to be elucidated. In the present study, a systems pharmacology model, which integrated cluster ligands, human intestinal absorption and aqueous solution prediction, chemical space mapping, molecular docking and network pharmacology techniques were used. The compounds from DSMF were diverse in the clusters and chemical space. The majority of the compounds exhibited drug-like properties. A total of
\end{abstract}

Correspondence to: Professor Xian-Xiang Liu, Institute of Bone Disease, Academy of Integrative Medicine, Fujian University of Traditional Chinese Medicine, 1 Qiuyang road, Fuzhou, Fujian 350122, P.R. China

E-mail: liuxianxiangfz@163.com

*Contributed equally

Abbreviations: DSMF, Diesun Miaofang; AS, Angelica sinensis; $R R$, Radix rehmanniae; AC, Areca catechu; RPR, Radix paeoniae rubra; TCM, Traditional Chinese Medicine; HIA, human intestinal absorption; PDE5A, phosphodiesterase 5A; PDE4A, phosphodiesterase 4A; ACE, angiotensin-converting enzyme; COMT, catechol O-methyltransferase; PNMT, phenylethanolamine $\mathrm{N}$-methyltransferase; IKK, inhibitor of $\kappa \mathrm{B}$ kinase; JNK, c-Jun terminal kinase; COX-2, cyclooxygenase-2; PLA2, phospholipase A2; LTA4H, leukotriene A-4 hydrolase; TNF- $\alpha$, tumor necrosis factor- $\alpha$; TP, thromboxane A2 receptor; MW, molecular weight; nHDon, number of HBond donors; nHAcc, number of HBond acceptors; AlogP, octanol-water partition coefficients; Std Dev, standard deviation

Key words: traditional Chinese medicine, Diesun Miaofang, traumatic injury, polypharmacology, systems pharmacology
59 compounds were identified to interact with 16 potential targets. In the herb-compound-target network, the majority of compounds acted on only one target; however, a small number of compounds acted on a large number of targets, up to a maximum of 12 . The comparison of key topological properties in compound-target networks associated with the above efficacy intuitively demonstrated that potential active compounds possessed diverse functions. These results successfully explained the polypharmcological mechanism underlying the efficiency of DSMF for the treatment of traumatic injury as well as provided insight into potential novel therapeutic strategies for traumatic injury from herbal medicine.

\section{Introduction}

Traumatic injury is one of the most common diseases in orthopedics. Its manifestations predominantly include localized pain, purplish swelling and joint dysfunction. Traumatic injury may also affect diet and sleep. The primary western drugs clinically available are analgesics and anti-inflammatory drugs, which predominantly alleviate the pain and swelling; however, these drugs have numerous side-effects (1). Recovery from traumatic injury is slow; therefore, it is essential to develop novel drugs suitable for prolonged use.

Diesun Miaofang (DSMF), a well-known herbal formula developed in the Ming Dynasty, has been widely used to treat traumatic injuries associated with parts of the body in China (2). The major herbs of this formula are Angelica sinensis (AS), Radix rehmanniae $(R R)$, Areca catechu $(A C)$ and Radix paeoniae rubra $(R P R)$; these herbs have been reported to work together to activate blood, remove stasis, promote qi circulation and relieve pain (3). However, the molecular features and active components of DSMF, as well as how these active components exert effects on targets remain to be elucidated. Several computer simulation methods have been successfully used to understand the theory of Traditional Chinese Medicine (TCM) and the relevant mechanisms of action from the molecular and systems level (4-8). In the present study, it 
was hypothesized that an integrated model combining cluster ligands, human intestinal absorption and aqueous solution prediction, chemical space mapping, molecular docking and network pharmacological techniques, may shed light on the herbal molecular features and pharmacological information of DSMF. These attempts offer key information for drug development from TCM and uncover holistic and synergic essence for TCM, which provided a guide for the modernization of TCM.

\section{Materials and methods}

Molecular database building and compound clustering. Chemical ingredients from $A S, R R, A C$ and $R P R$ were collected from the Chinese Herbal Drug Database (2002 version) and the Handbook of the Constituents in Chinese Herb Original Plants $(9,10)$. Excluding duplicates, 158 compounds were determined and their structures were drawn using ISIS Draw 2.5 (MDL Information Systems, Inc., San Leandro, CA, USA), then further optimized using Discovery studio 2.0 (DS2.0; Accelrys, Inc., San Diego, CA, USA) with a Merck molecular force field (Merck Research Laboratories, Boston, MA,USA). The protocol of cluster ligands in DS2.0 were performed to group DSMF compounds under the standard default settings (11).

Human intestinal absorption (HIA) and aqueous solubility prediction. HIA following oral administration and aqueous solubility were predicted using the ADMET module of DS2.0 (12). Compounds with an absorption level of $0,1,2$ or 3 were considered to have good, moderate, poor or very poor absorption, respectively. Compounds with the solubility levels in the interval from 2 to 4 were considered to exhibit drug-like properties. Acceptable HIA means HIA level is 0 or 1 , and acceptable solubility means solubility level values are 2, 3 or 4 .

Chemical space mapping and analysis. In order to investigate whether the four herbs had diverse chemical components, 150 physicochemical properties, including 1 dimensional (D)-, 2D- and 3D-molecular descriptors, were selected for analysis using the Quantitative Structure-Activity Relationship module of DS2.0 (6,13). Distribution of chemical ingredients in the chemical space was visualized via principal component analysis using the library analysis module of DS2.0. In addition, analysis of four important pharmacology-associated descriptors, including molecular weight (MW), number of HBond donors (nHDon), number of HBond acceptors (nHAcc) and octanol-water partition coefficients $(\mathrm{A} \log \mathrm{P})$, were performed to predict the drug-likeness of DSMF base on Sigmaplot version 10.0 (Systat Software, Inc., San Jose, CA, USA).

Potential active compound prediction. Molecular docking was performed to determine whether DSMF interacted with the key proteins associated with blood circulation activation and anti-inflammation, using the LigandFit module of DS2.0. The crystal structures of protein-ligand complexes were downloaded from the Research Collaboratory for Structural Bioinformatics Protein Data bank (http://www. pdb.org/; Table I), with the exception of thromboxane A2 receptor (TP) (14-17). The homology model of TP was obtained from the Center for Experimental Therapeutics and Pharmacoinformatics (University of Houston, Houston, TX,
Table I. Key target proteins associated with activating blood, removing stasis, promoting qi circulation and relieving pain.

A, Targets associated with activating blood and removing stasis

\begin{tabular}{ll}
\hline Protein & PDB code \\
\hline Thrombin & $1 \mathrm{AWH}$ \\
Factor-IXa & $1 \mathrm{RFN}$ \\
PDE5A & $4 \mathrm{G} 2 \mathrm{~W}$ \\
ACE & $1 \mathrm{O} 66$ \\
ACE2 & $1 \mathrm{R} 42$ \\
Catechol-O-methyl transferase & $3 \mathrm{BWM}$ \\
Phenylethanolamine N-methyltransferase & $2 \mathrm{AN} 4$ \\
Abelson tyrosine-protein kinase1 & $3 \mathrm{QRI}$ \\
\hline
\end{tabular}

B, Targets associated with promoting qi circulation and relieving pain

\begin{tabular}{lc}
\hline Protein & PDB code \\
\hline Inhibitor of nuclear factor $\kappa-B$ kinase- $\beta$ & $3 \mathrm{RZF}$ \\
JNK-1 & $2 \mathrm{GMX}$ \\
JNK-3 & $1 \mathrm{PMN}$ \\
P38 & $1 \mathrm{~A} 9 \mathrm{U}$ \\
Cyclooxygenase-2 & $6 \mathrm{COX}$ \\
Phospholipase 2 & $1 \mathrm{DB} 4$ \\
Leukotriene A4 hydrolase & $3 \mathrm{~B} 7 \mathrm{R}$ \\
Tumor necrosis factor- $\alpha$ & $2 \mathrm{AZ5}$ \\
PDE4A & $3 \mathrm{TVX}$
\end{tabular}

PDB, protein data bank; PDE, phosphodiesterase; ACE, angiotensin-converting enzyme; JNK, c-Jun N-terminal kinases; TNF, tumor necrosis factor.

Table II. Cluster result of compounds from Diesun Miaofang.

\begin{tabular}{lcl}
\hline Cluster & Number of compounds & \multicolumn{1}{c}{ Source } \\
\hline 1 & 7 & $A S, R R, A C$ \\
2 & 13 & $A S, A C$ \\
3 & 34 & $A S, R R, A C$ \\
4 & 28 & $R R, A C, R P R$ \\
5 & 2 & $R R$ \\
6 & 2 & $A S$ \\
7 & 2 & $A C$ \\
8 & 14 & $A S, R R, A C, R P R$ \\
9 & 13 & $A S, R R, A C$ \\
10 & 43 & $R R, R P R$ \\
\hline
\end{tabular}

AS, Angelica sinensis; RR, Radix rehmanniae; AC, Areca catechu; RPR, Radix Paeoniae Rubra.

USA) $(18,19)$. For each crystal structure, crystallographic water molecules were removed and missing hydrogen atoms were added. The inhibitor from the crystal structure defined 
Table III. Key molecular properties of compounds in Diesun Miaofang.

\begin{tabular}{lcccc}
\hline Property & Mean & $\begin{array}{c}\text { Standard } \\
\text { deviation }\end{array}$ & Minimum & Maximum \\
\hline Number of carbon atoms & 16.67 & 8.97 & 4 & 41 \\
Number of nitrogen atoms & 0.16 & 0.79 & 0 & 8 \\
Number of oxygen atoms & 6.66 & 6.33 & 0 & 26 \\
Molecular weight & 334.78 & 205.36 & 94.11 & 940.68 \\
Number of hydrogen acceptors & 6.78 & 6.31 & 0 & 26 \\
Number of hydrogen donors & 3.78 & 3.91 & -9.55 & 17 \\
Octanol-water partition coefficients & 1.07 & 3.45 & & 9.13 \\
\hline
\end{tabular}

Table IV. Simple parameters of H-C-T and C-T networks.

\begin{tabular}{lccc}
\hline & & \multicolumn{2}{c}{ C-T network function } \\
\cline { 3 - 4 } Parameter & $\begin{array}{c}\text { H-C-T } \\
\text { network }\end{array}$ & $\begin{array}{c}\text { Activating blood and } \\
\text { removing stasis }\end{array}$ & $\begin{array}{c}\text { Promoting qi circulation } \\
\text { and relieving pain }\end{array}$ \\
\hline Network centralization & 0.423 & 0.109 & 0.110 \\
Network heterogeneity & 0.911 & 0.891 & 0.926 \\
Characteristic path length & 2.821 & 3.924 & 3.372 \\
Average number of neighbors & 5.012 & 2.880 & 2.939 \\
\hline
\end{tabular}

H-C-T, herb-compound-target; C-T, compound-target.

Table V. Key compounds in the herb-compound-target network.

\begin{tabular}{crl}
\hline Degree & Index & \multicolumn{1}{c}{ Chemical name } \\
\hline 12 & 12 & Proanthocyanidin B2 \\
8 & 18 & Eugeniin \\
7 & 146 & Rehmannioside A \\
7 & 110 & Isoacteoside \\
6 & 25 & (Z)-(1S,5R)- $\beta$-Pinen- \\
& & 10-yl- $\beta$-vicianoside \\
6 & 4 & Catechin \\
5 & 78 & Adenosine \\
5 & 57 & Ferulic acid \\
4 & 157 & 6-O-Vanilloylajugol \\
4 & 20 & Oxypaeoniflorin \\
\hline
\end{tabular}

the active site. A total of 158 DSMF compounds were docked into the protein models and interactions between them were evaluated by DockScore which estimates the ligand position and orientation based on the most favorable energy production from the interactions between the ligand conformations and receptor proteins (20). Compounds with top 8 DockScores were selected as potential active compounds in DSMF (21).

Network construction and analysis. To elucidate the functional mechanism underlying DSMF, the compound-target (C-T)

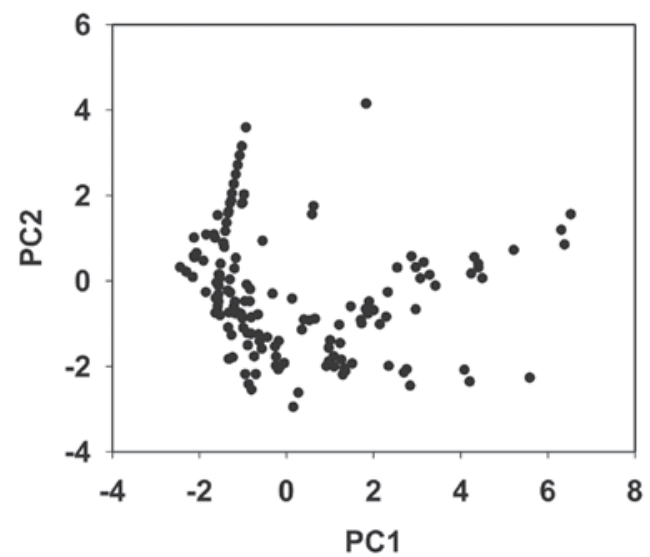

Figure 1. Chemical space distribution of compounds in Diesun Miaofang.

network and herb-compound-target (H-C-T) network were constructed. The procedure for network construction was as follows: The C-T network was constructed by linking the potential active compounds and their corresponding targets; and the H-C-T network was constructed by connecting previously mentioned compounds to any associated herbs. All networks were produced and analyzed using Cytoscape 2.8.3 (University of California, San Diego, CA, USA) (22).

\section{Results}

Molecular features of DSMF. The compounds from DSMF were divided into 10 clusters (Table II). These compounds 

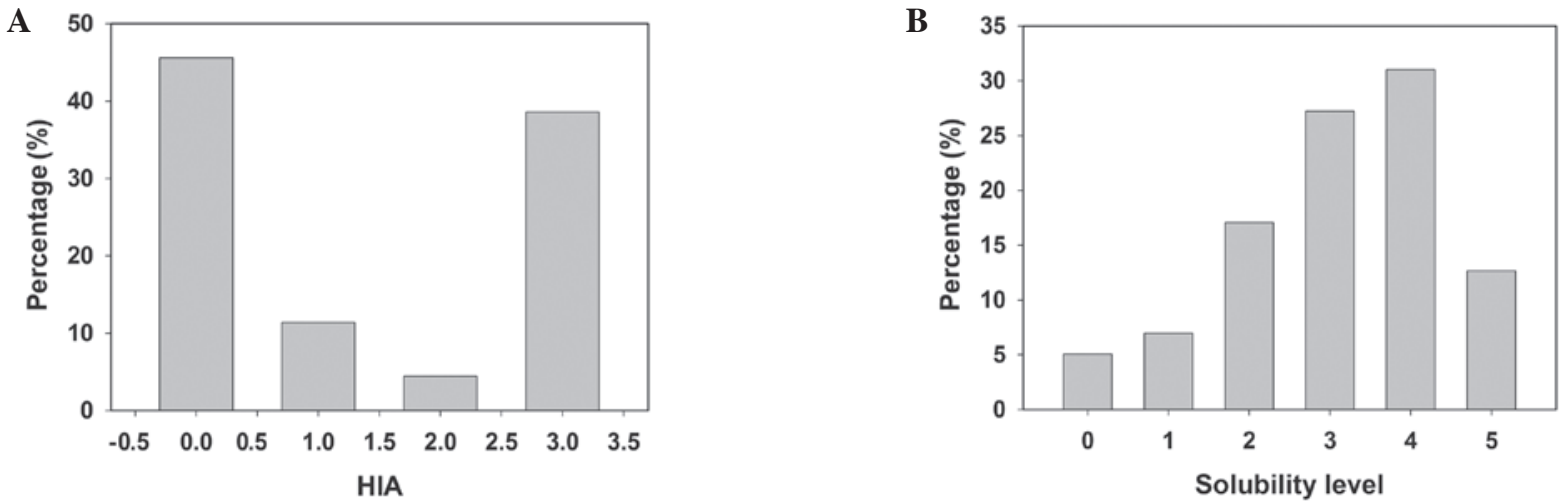

Figure 2. (A) Distributions of HIA and (B) aqueous solubility of compounds from Diesun Miaofang. HIA, human intestinal absorption.

A
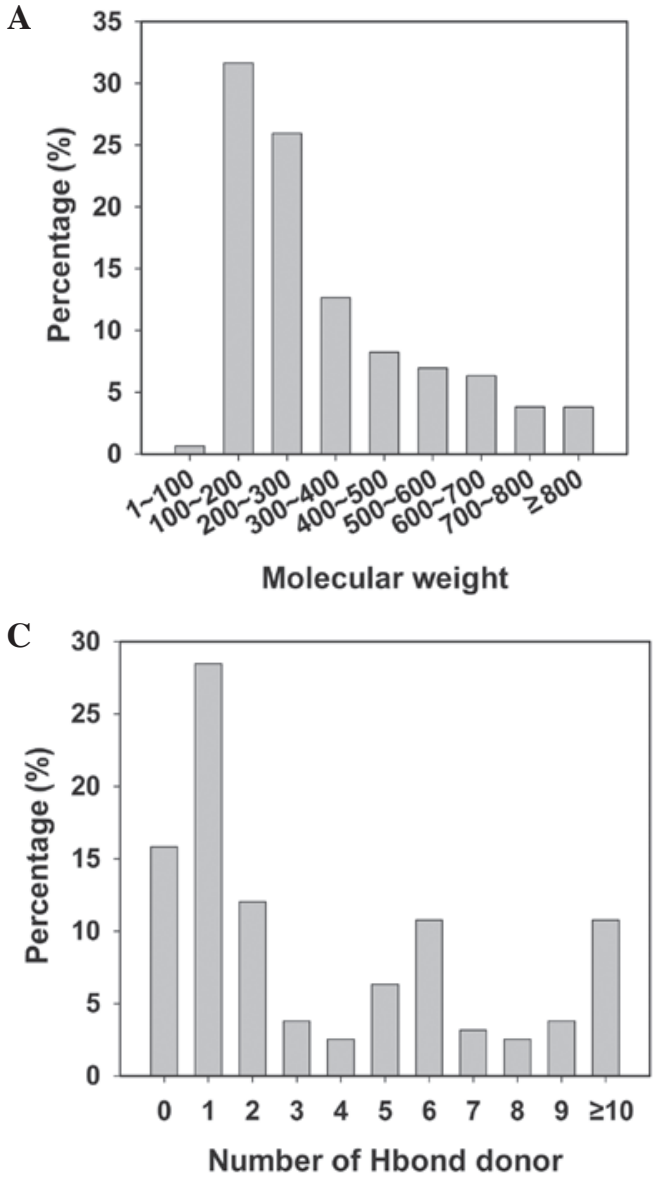

B

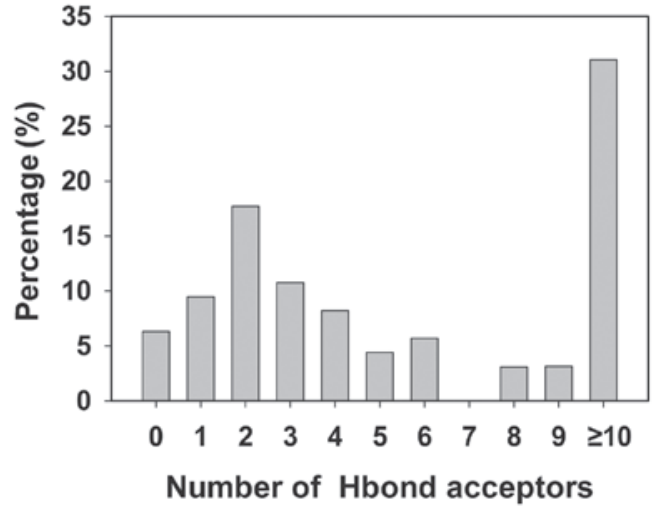

D

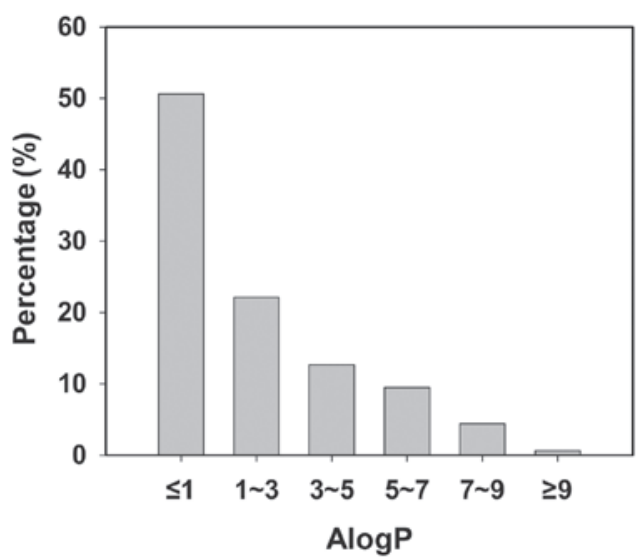

Figure 3. Profile distributions of four important molecular properties of compounds from Diesun Miaofang. The molecular properties consist of the molecular (A) weight, (B) number of HBond acceptors, (C) number of HBond donors and (D) AlogP. AlogP, octanol-water partition coefficients.

were widely distributed in the chemical space (Fig. 1; Table III). Additionally, $>56 \%$ exhibited an acceptable HIA and aqueous solubility (Fig. 2). Four molecular descriptors, including MW, nHAcc, nHDon and AlogP, which reflect the basic characteristics of drug-likeness were analyzed and are shown in Fig. 3. The percentages of compounds with MW $<500 \mathrm{Da}$, nHAcc $<10$, nHDon $<5$ and AlogP $<5$ were 79.12, $69.62,62.66$ and $85.44 \%$, respectively. These results indicated that the compounds from DSMF were diverse and exhibited potent drug-like properties for oral administration, according to the 'rule of five' (23).
Potential active compounds and diverse functions in DSMF. A total of 59 compounds were screened out by the docking method, which linked with compounds with 16 targets. To highlight the functions of DSMF, one H-C-T network and two C-T networks associated with activating blood, removing stasis, promoting qi circulation and relieving pain were constructed and are shown in Figs. 4-6. In Fig. 4, 29 compounds were associated with only one target, while 30 compounds of exhibited a relatively potent interaction with $\geq 2$ targets. Notably, six compounds were associated with $>5$ targets. The key network parameters of the above networks and compounds in the H-C-T network were listed in 


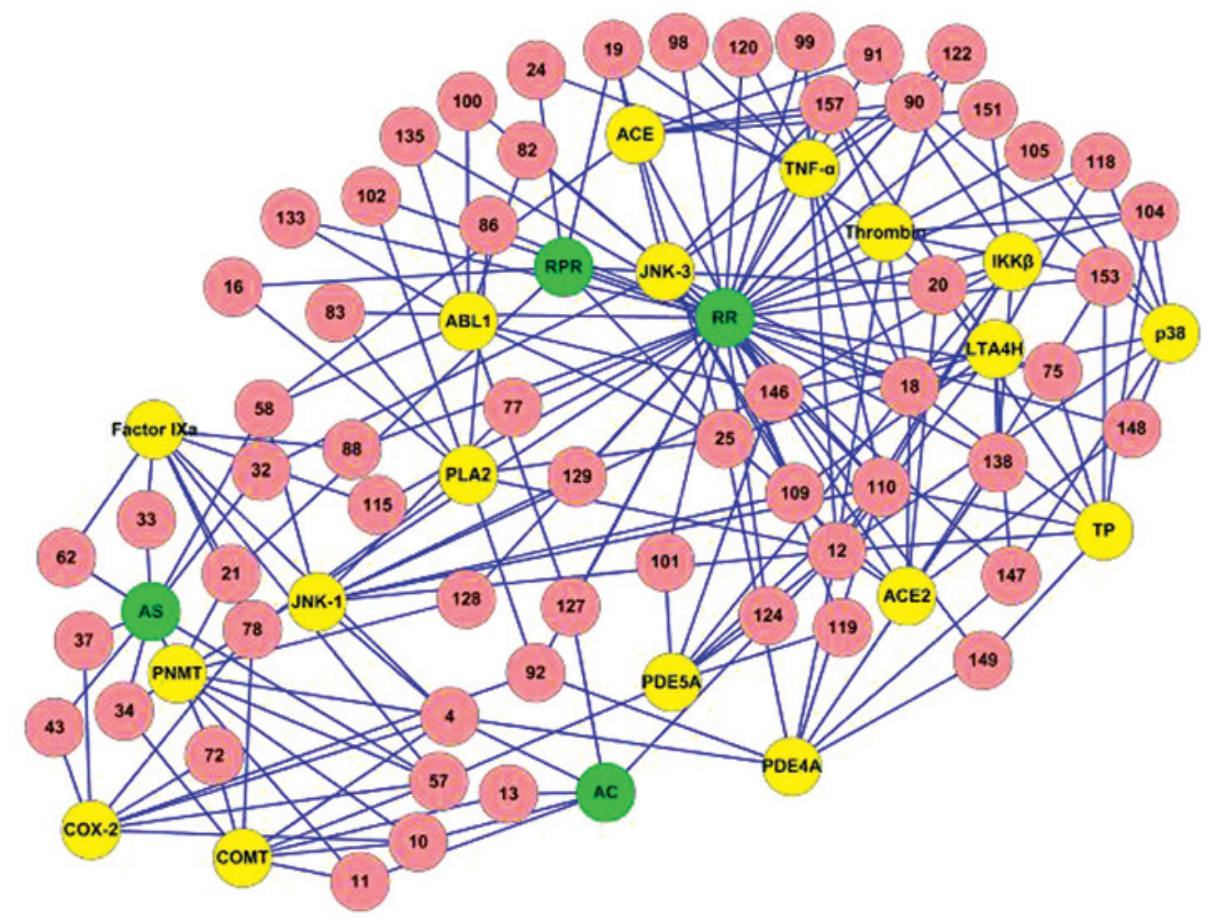

Figure 4. Herb-compound-target network. Green, pink and yellow circles represent the herbs, compounds and targets, respectively.

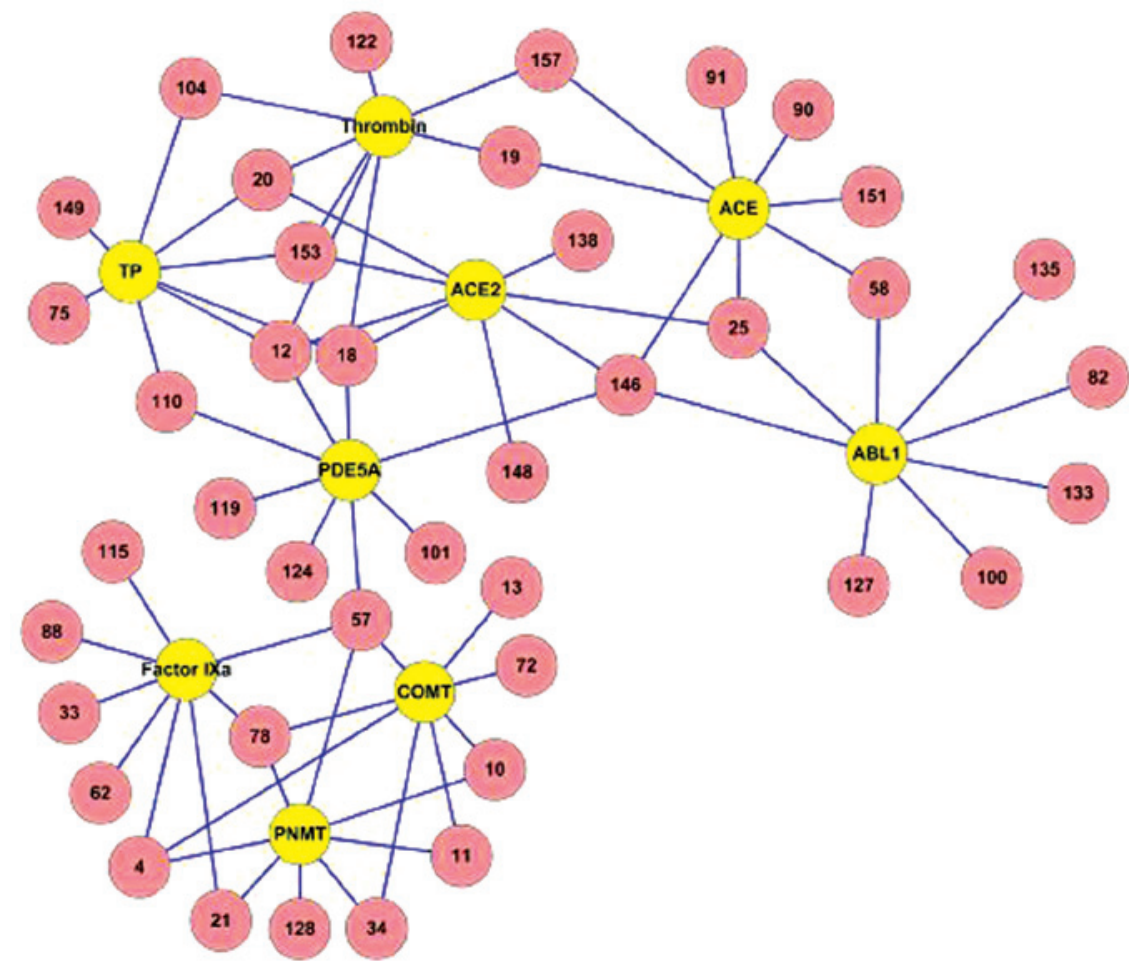

Figure 5. Compound-target network associated with activating blood and removing stasis. Pink and yellow circles represent compounds and targets, respectively.

Tables IV and V, respectively. The topological properties of two C-T networks were analyzed in Fig. 7. The two C-T networks with different functions had a small number of overlaps in the distributions of betweenness centrality, closeness centrality, average shortest path length and topological coefficients, which indicated that certain compounds in the two networks may have synergy, and more compounds may possess diverse functions.

\section{Discussion}

TCM, as an important part of complementary and alternative medicine, demonstrated an improved effect on osteopathia (24). However, the active substances involved in the herbal formulae and the mechanisms of action associated with the therapeutic effectiveness remain to be fully elucidated. Additionally, 


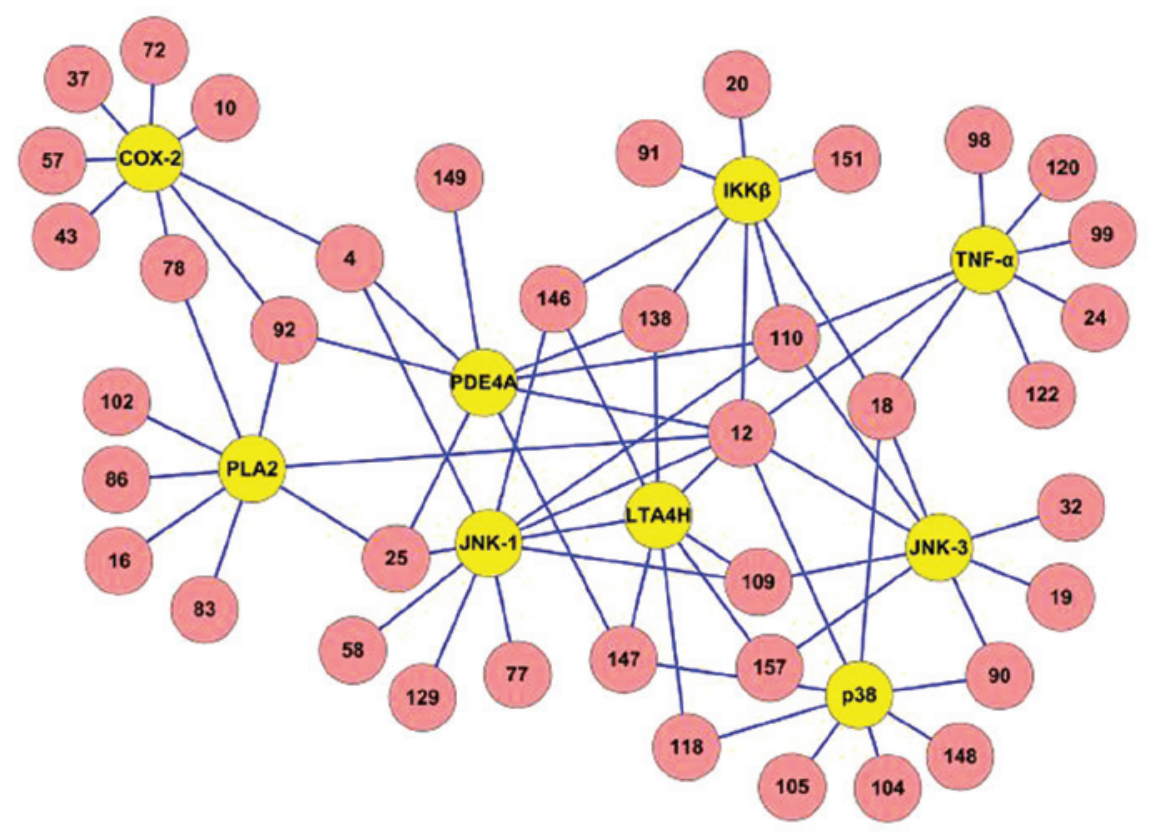

Figure 6. Compound-target network associated with promoting qi circulation and relieving pain. Pink and yellow circles represent compounds and targets, respectively.

A

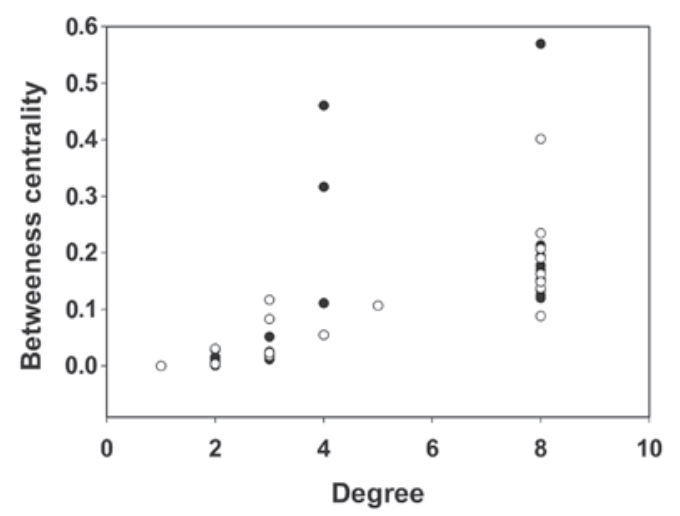

C

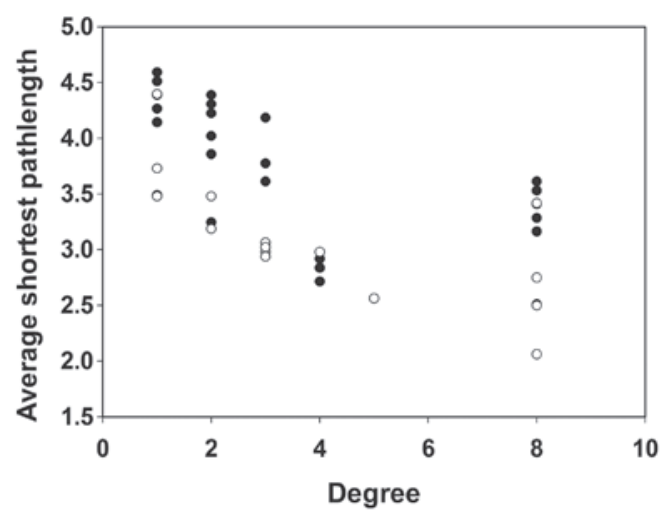

B

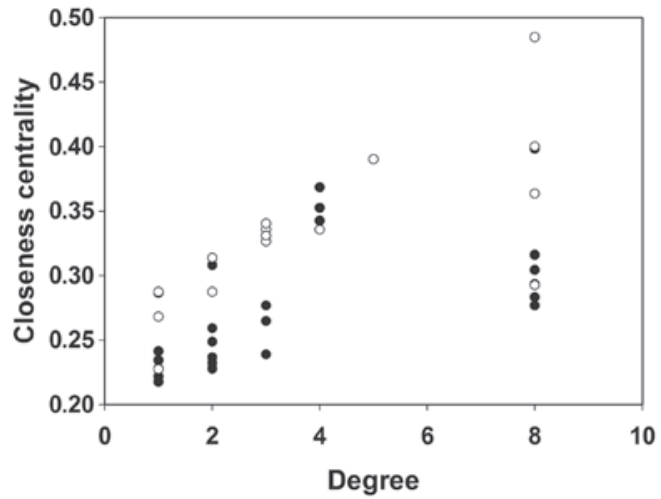

D

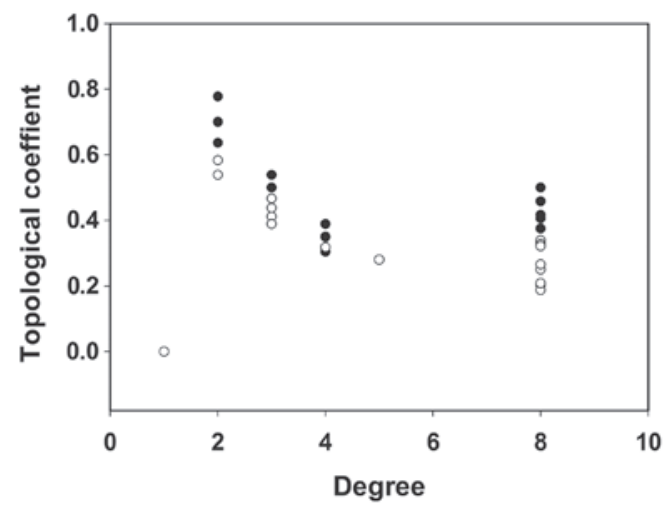

Figure 7. (A-D) Profile distributions of key topological properties for two compound-target networks. Black circles represent the topological properties of the compound-target network associated with activating blood and removing stasis and white circle represent those of the compound-target network associated with promoting qi circulation and relieving pain.

conventional methods of herbal investigation generally focus on extraction, isolation, purification, structure identification and activity assays. These often lead to a failure to screen out certain active ingredients from herbs and may not reflect the integrality of TCM (25). Therefore, determining reasonable approaches to solve the above shortcomings has become a focus of research $(26,27)$.

There has been an increasing focus on identifying novel drugs from TCM and elucidating the underlying pharmacological mechanisms of TCMs via different modern computational 
pharmacological methods (28). These are important for improving current understanding of the nature of TCM. In the present study, Table II demonstrated that the compounds from DSMF may be separated into 10 independent clusters. The compounds from clusters 1,2,3, 4, 8, 9 and 10, were observed in no less than two herbs, suggesting that different herbs may have similar structural features. The clusters 5,6 and 7 also suggested that different herbs may have their own structural features. In order to gain more knowledge regarding the physicochemical features of compounds from DSMF, the chemical space of DSMF was mapped in Fig. 1. According to the theory of chemical space (13), the superposed and close dots indicated that these compounds may have similar mechanisms of action and may act on identical targets. Conversely, dispersive dots indicated that these compounds may have different pharmacological activities. These results may explain why DSMF has diverse efficacies from TCM theory. In addition, Figs. 2 and 3 demonstrated that the compounds from DSMF have favorable drug-like properties and are suitable for screening lead compounds.

Docking results revealed that 59 potential compounds exhibited desired interactions with the targets. The global understanding of the association among the herbs, compounds and targets was shown in Fig. 2. Out of the 59 potential compounds, 30 had at least two links with other targets, indicating that the majority of targets share common compounds with other targets. The average number of potential targets per compound was 3.28. In addition, the four herbs from DSMF were found to work together on 9 common targets, suggesting that DSMF was a broad-spectrum formula acting on multiple targets. Since the synergistic multitarget effects mean that natural products affect not only one target, but several targets (5), DSMF has potential synergistic interactions.

To further elucidate the multicompound therapeutic mechanism of DSMF in the present study, the key topological properties of two compound-target networks associated with activating blood, removing stasis, promoting qi circulation and relieving pain, including betweenness centrality, closeness centrality, average shortest path length and topological coefficient, were selected to perform the analysis (Figs. 5, 6 and 7). These results may provide important information with regards to the properties of compounds and proteins involved in the network. As shown in Fig. 5, the efficacy of activating blood and removing stasis may be accomplished by interacting with several targets associated with hemostasis and blood circulation. Fig. 6 also demonstrated that DSMF may promote qi circulation and relieve pain by influencing several targets associated with anti-inflammation. For example, ferulic acid in Table V presents a relatively strong interaction with these targets associated with hemostasis and blood circulation (catechol-O-methyl transferase, phenylethanolamine N-methyltransferase, phenylethanolamine, phosphodiesterase 5A and Factor-IXa) and anti-inflammation (cyclooxygenase-2). Notably, it has been reported that ferulic acid had effects on anti-platelet aggregation and alleviating pain $(29,30)$. In addition, in the present study, Fig. 7 demonstrated that certain nodes with an identical degree may have different effects on the above topological properties, which suggested that there are different modes of action between compounds and targets during the treatment of DSMF. Taken together, these results consistently indicated that
DSMF exists as multicompounds and combination therapies to act on several targets, finally exerting diverse pharmacological effects on traumatic injury.

In conclusion, the present study provided a systems pharmacology model to dissect the molecular features and modes of action of DSMF. The key findings demonstrated that the compounds from DSMF were diverse in chemical structure and widely distributed in chemical space, the majority of the compounds from DSMF had potent drug-like properties for oral administration and DSMF possessed multicompound synergy associated with the interacting targets and exerted different pharmacological effects. The present study provided a systematic view of the association between components in herbal formula, the efficacy of herbal formula and targets, which contributed to an improved understanding of the chemical and pharmacological basis of TCM, which may enhance the process of drug discovery from TCM.

\section{Acknowledgements}

The present study was supported by a grant from the Developmental Fund of ChenKeji Integrative Medicine (no. CKJ2010032).

\section{References}

1. Quan LG, Liu H and Wang JY: The effect of external application of herbal medicines on 1147 patients with swelling and pain after traumatic injury. Chinese Journal of Rural Medicine 8: 39-40, 2010 (In Chinese)

2. Luo YW and Sun ZG: The brief analysis of treatment in line with the seasons, change of climate in Diesun Miaofang. Journal of Hunan College of Traditional Chinese Medicine 15: 12-14, 1995 (In Chinese).

3. Li JM and Li X, (eds): The application methods of drug and commonly used formulae of traditional Chinese traumatology. People's Military Medical Press, Beijing, pp104-113, 1994 (In Chinese).

4. Zheng CS, Xu XJ, Ye HZ and Liu XX: Comparative Research on computer simulation of two different therapeutic principles and formulae for osteoarthritis. Zhongguo Zhong Xi Yi Jie $\mathrm{He} \mathrm{Za}$ Zhi 34: 480-485, 2014 (In Chinese).

5. Liu H, Wang J, Zhou W, Wang Y and Yang L: Systems approaches and polypharmacology for drug discovery from herbal medicines: an example using licorice. J Ethnopharmacol 146: 773-793, 2013.

6. Zheng CS, Xu XJ, Ye HZ, Wu GW, Li XH, Huang SP and Liu XX: Computational approaches for exploring the potential synergy and polypharmacology of Duhuo Jisheng Decoction in the therapy of osteoarthritis. Mol Med Rep 7: 1812-1818, 2013.

7. Shi SH, Cai YP, Cai XJ, Zheng XY, Cao DS, Ye FQ and Xiang Z: A network pharmacology approach to understanding the mechanisms of action of traditional medicine: Bushenhuoxue formula for treatment of chronic kidney disease. PLoS One 9: e89123, 2014.

8. Zheng CS, Ye HZ, Xu XJ and Liu XX: Computational pharmacology study of tougu xiaotong granule in preventing and treating knee osteoarthritis. Chin J Integr Med 15: 371-376, 2009 (In Chinese).

9. Qiao X, Hou T, Zhang W, Guo S and Xu X: A 3D structure database of components from Chinese traditional medicinal herbs. J Chem Inf Comput Sci 42: 481-489, 2002.

10. Zhou JX, Xie GR and Yang XD: Handbook of the chemical constituents in chinese herb original plants. Chemical industry Press, Beijing, pp1165-1211, 2004 (In Chinese).

11. Hassan M, Bielawski JP, Hempel JC and Waldman M: Optimization and visualization of molecular diversity of combinatorial libraries. Mol Divers 2: 64-74, 1996.

12. Zheng CS, Xu XJ, Liu XX and Ye HZ: Computational pharmacology of Jingzhi Tougu Xiaotong granule in preventing and treating Osteoarthritis. Acta Phys Chim Sin 26: 775-783, 2010 (In Chinese). 
13. Dobson CM: Chemical space and biology. Nature 432: 824-828, 2004.

14. Zhou W and Wang Y: A network-based analysis of the types of coronary artery disease from traditional Chinese medicine perspective: potential for therapeutics and drug discovery. J Ethnopharmacol 151: 66-77, 2014.

15. Liu HB, Xu J, Peng Y, Zhou JX and Xiao PG: Targets of Danshen's active components for activating circulation activities. Acta Phys Chim Sin 26: 199-205, 2010 (In Chinese).

16. Liu ZZ, Liang JP, Nie YC, Liu H, Xie CS, Cheng GH and Su WW: Network pharmacology study of compound xueshuantong capsule based on the targets related to blood circulation and hemostasis. Acta Scientiarum Naturalium Universitatis Sunyatseni 52: 97-100, 2013 (In Chinese).

17. Zhang H, Zan JH, Long W and Liu PX: Study on the anti-inflammatory mechanism of Chinese medicine herbs by the molecular docking method. Heralad Med 31: 1542-1546, 2012 (In Chinese)

18. Ruan KH, Wijaya C, Cervantes V and Wu J: Characterization of the prostaglandin $\mathrm{H} 2$ mimic: binding to the purified human thromboxane A2 receptor in solution. Arch Biochem Biophys 477: 396-403, 2008.

19. Wu J, Feng M and Ruan KH: Assembling NMR structures for the intracellular loops of the human thromboxane A2 receptor: implication of the $\mathrm{G}$ protein-coupling pocket. Arch Biochem Biophys 470: 73-82, 2008.

20. Montes M, Braud E, Miteva MA, Goddard ML, Mondésert O, Kolb S, Brun MP, Ducommun B, Garbay C and Villoutreix BO: Receptor-based virtual ligand screening for the identification of novel CDC25 phosphatase inhibitors. J Chem Inf Model 48: 157-165, 2008

21. Zheng CS, Ye HZ, Cai LL, Chen JS, Wei LS and Liu XX: Discussion on multi-component and multi-target pattern of Liuwei Dihuang pill in the treatment of osteoarthritis on the basis of computer simulation. J Trad Chin Orthop Traumatol 25: 11-13, 2013 (In Chinese).
22. Smoot ME, Ono K, Ruscheinski J, Wang PL and Ideker T: Cytoscape 2.8: new features for data integration and network visualization. Bioinformatics 27: 431-432, 2011.

23. Lipinski CA, Lombardo F, Dominy BW and Feeney PJ: Experimental and computational approaches to estimate solubility and permeability in drug discovery and development settings. Adv Drug Deliv Rev 46: 3-26, 2001.

24. Chen MC: Effect of fumigation therapy for ankle injury. J Nurs Sci 20: 28-29, 2005

25. Xu X: New concepts and approaches for drug discovery based on traditional Chinese medicine. Drug Discov Today Technol 3: 247-253, 2006.

26. Li S and Zhang B: Traditional Chinese medicine network pharmacology: Theory, methodology and application. Chin J Nat Med 11: 110-120, 2013.

27. Xu XJ: Study on computer simulation for Chinese traditional compound medicine. Prog Chem 11: 202-204, 1999 (In Chinese).

28. Li P, Chen J, Wang J, et al: Systems pharmacology strategies for drug discovery and combination with applications to cardiovascular diseases. J Ethnopharmacol 151: 93-107, 2014.

29. Xu Y,Zhang L, Shao T, et al: Ferulic acid increases pain threshold and ameliorates depression-like behaviors in reserpine-treated mice: behavioral and neurobiological analyses. Metab Brain Dis 28: 571-583, 2013.

30. Zhao J, Suyama A, Tanaka M and Matsui T: Ferulic acid enhances the vasorelaxant effect of epigallocatechin gallate in tumor necrosis factor-alpha-induced inflammatory rat aorta. J Nutr Biochem 25: 807-814, 2014. 